\title{
Tessella-oriented Segmentation and Guidelines Estimation of Ancient Mosaic Images
}

\author{
Lamia Benyoussef and Stéphane Derrode \\ GSM Group, Institut Fresnel (CNRS UMR 6133) \& École Centrale Marseille, \\ Technopôle de Château-Gombert, 8, rue Frédéric Joliot Curie, \\ 13451 Marseille Cedex 20, France. \\ firstname.lastnamedec-marseille.fr
}

August 28, 2008

\begin{abstract}
Automatic segmentation and analysis of ancient mosaic images can help archeologists and experts build digital collections and automatically compare mosaics by means of image database indexing and content-based retrieval tools. However, ancient mosaics are characterized by low contrast colors, irregular tessella shape, orientation and positioning, making automatic segmentation difficult. In this work we propose a tessella-oriented strategy whose first step consists in isolating tessellas from its cemented network by computing the watershed transformation of a criterion image generated to exhibit the cement network as watershed crests. Then a simple k-means algorithm is used to classify tessellas and segment mosaic images with more accuracy than with a pixeloriented strategy. Additionally, we propose a method to automatically get the main directional guidelines of mosaics by estimating tessella orientation. This is done by minimizing a contextual energy computed from gray-level means of neighboring tessellas and orientation of their borders. Several examples of cartographies show the effectiveness of the method.
\end{abstract}


Keywords: Ancient mosaic image, Mosaic segmentation, Mosaic directional guidelines.

\section{INTRODUCTION}

The aim of this work is to (1) analyse ancient mosaic images (2) characterize their structure and color by means of automatic processing tools. The final goal is to detect and localize objects with a semantic meaning like animal, human, object etc. in a complex mosaic scene. This can help archeologists and experts in their historical and artistic studies, especially the analysis of ancient mosaicist styles (i.e. opus musivum and opus vermiculatum* ${ }^{*}$. Such tools can also be of interest for (i) museums in order to categorize mosaics and to draw up a digital inventory of their collection ${ }^{\dagger}$ and (ii) computed-aided generation of old-style mosaic images from a master image ( $c f$. [1] for an overview of digital mosaic frameworks and references cited therein). A first attempt to propose a contentbased and image retrieval system dedicated to ancient mosaic images has been presented in [2]. In this work, efforts have been focused on pattern recognition aspects, by using an invariant description of semantic objects present in scenes using Fourier-Mellin transform [3,4]. Semantic objects are isolated using statistical segmentation and morphological operators, but extraction remains a difficult task inherent to the way mosaics are built.

Mosaics are made of colored tiles, called tessera or tessella, usually formed in the shape of a cube of materials separated by a cement joint. Smart and

\footnotetext{
* Mosaic Art in Vitreous Glass, Millefiori, Tesserae Mosaics by Shelby Glass Studio, www.mosaic-tileart.com/mosaic.html, 2006.

†Examples: Musée des Antiquités Nationales, Saint-Germain-en-Laye, France. www.culture.gouv.fr/culture/arcnat/vienne/en/. Musée National du Bardo, Tunisia, www.di . com.tn/museebardo/.
} 

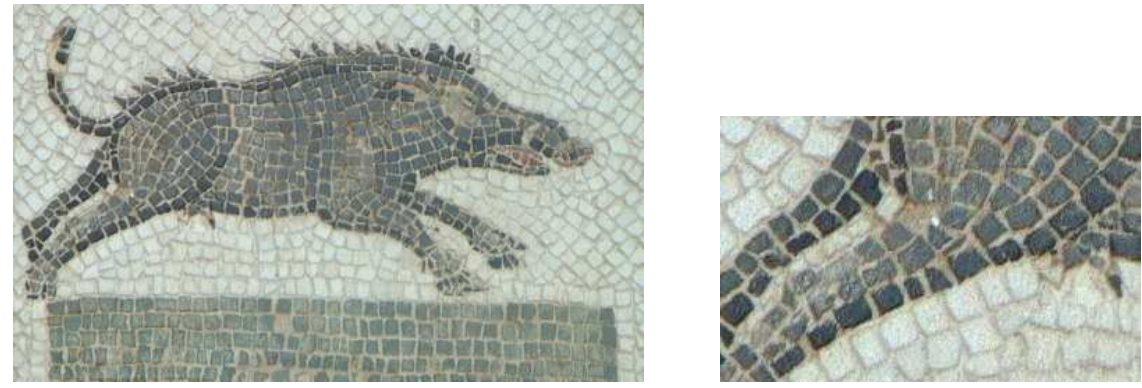

Fig. 1. Excerpt of an ancient mosaic showing a boar with a zoom on its hind legs. This image will be used later as a guiding thread for algorithm illustration.

judicious use of orientation, shape and size of tessellas characterize the artwork style and exhibit the "general flow" of the mosaic chosen by the mosaicist. Fig. 1 shows a typical example of a mosaic image to be processed. This kind of images shows specific difficulties inherent to their oldness and artwork style:

- Tessellas of ancient mosaics are characterized by pastel colors, with low contrast. Color information is not discriminant and gray-level values are generally sufficient to describe color dynamics in such an image.

- Shape of tessellas are irregular, from a square shape to a polygonal one. Their positioning and orientation are not aligned according to a rectangular grid.

- The positioning of tessellas makes the joint appear as an irregular network with numerous interconnections throughout the mosaic. Network intensity, mainly middle gray, is not uniform through the image because of tessella shadows due to non-flat mosaic surfaces and snapshot acquisition angle.

These particularities make segmentation methods based on pixel values inefficient. Indeed pixels associated to the cement network interfere and introduce confusions in the classification process. Hence, the strategy under which 
the work was conducted is to consider that tessellas are indivisible entities with almost a uniform gray-level value. So the first stage is to extract tessellas from the cement network. In Section 2, we present a strategy adapted to the mosaic network specificity. It is based on the watershed transformation of a particular criterion image built from the original image in order to exhibit the cement network as watershed crests. At this point, mosaic images are considered tessellaoriented and no more pixel-oriented, i.e. all processings are applied on tiles and not pixels. Hence, it was easy to obtain a robust segmentation of mosaic images by using a simple tessella-based k-means algorithm, which outperforms the classical pixel-based one.

Since orientation of tiles has a strong visual influence on the overall perception of the mosaic and also in order to facilitate delimitation of semantic objects in mosaic scene, we propose a simple and efficient way of estimating main orientations of tessellas (allowing to exhibit directional guidelines of mosaics) in ancient mosaic images in Section 3. The proposed approach consists in minimizing a contextual energy computed from mean-gray value of neighboring tessellas and orientation of their borders. Conclusions and further works are drawn in Section 4.

\section{TESSELLA EXTRACTION FOR MOSAIC SEGMENTATION}

The extraction of a network in an image is a recurrent problem, especially for road extraction from aerial photos $[5,6]$ or for vascular network segmentation from angiographies $[7,8]$. Several approaches have been proposed. Methods based on contour extraction are widely used and mainly rely on the assumption that the network pixels and neighboring ones have different gray levels in order to compute gradients. But methods based on high-pass filter, such as Har- 
ris's corner detector, highlight pixels belonging to the network, not connected components. Higher-level processings detect lines with varying widths $[9,10]$. Strategies that track the entire network from a starting point $[11,12]$ are difficult to justify in our case-study due to the high number of intersections in a typical mosaic network.

Numerous methods based on Markov modeling $[13,14]$ or active contours [15, 16] have also been proposed. These methods are quite efficient but time consuming. In the case of mosaics, these methods are not suited because of the high density of the network to be extracted in images. In [14], a Markov model is applied on a graph of adjacency crests, detected by a Watershed Transformation (WT) applied on a criterion image. This criterion image, computed from the original one, exhibits the potential of each pixel to belong to the network.

Among those methods, the WT approach appears interesting for mosaic images since this method is a good compromise between low-level methods (contour detection) and approaches by energy minimization (Markov model or active contours) which are unworkable due to cement network complexity in mosaic images. To work well, the WT needs to be computed on a criterion image that shows tessellas as catchment basins and the network as crests. But the network, mainly middle gray-valued, is sometimes darker than the tessellas and sometimes lighter in the same image. Hence, for each pixel in the image, we study the gray-level profile around it according to four directions $\left(0^{\circ}, 45^{\circ}, 90^{\circ}\right.$ and $135^{\circ}$ ) and compare them to two templates characteristic of the two situations, i.e. dark network and light tessellas and light network and dark tessellas. The value of a pixel in the criterion image is the minimum value among the eight ones. If this value is high then we face a somewhat flat profile that indicates a pixel inside a tessella. 


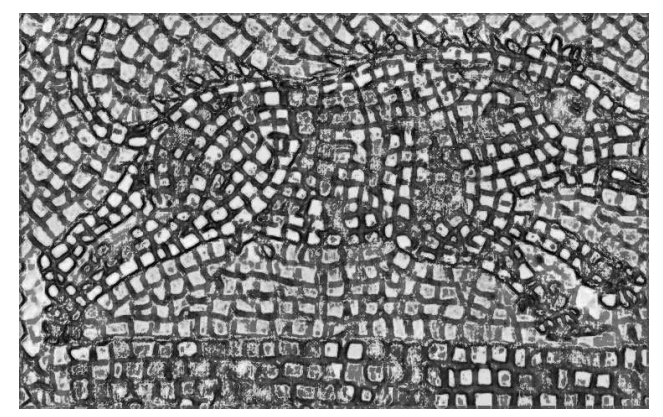

Fig. 2. Criterion image obtained from the boar image in Fig. 1.
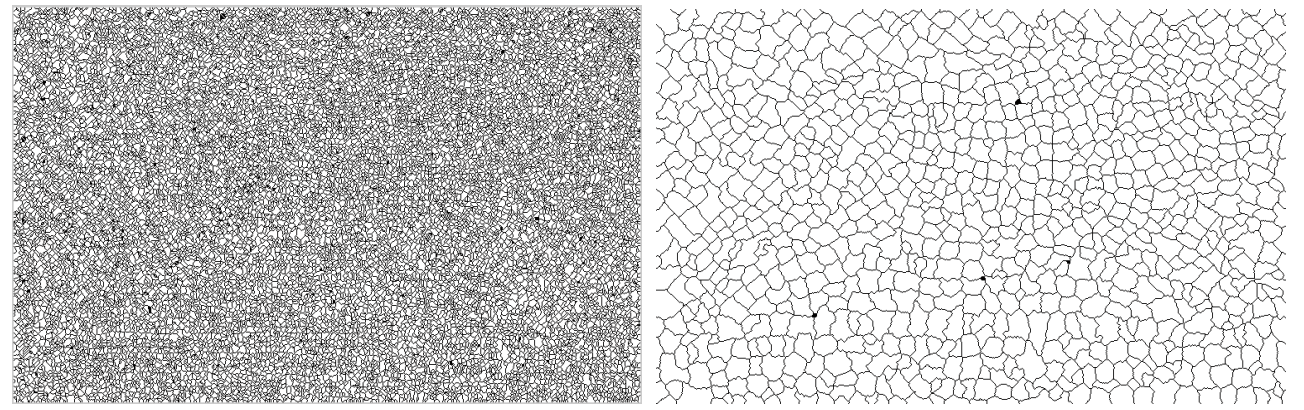

Fig. 3. Extraction of tessellas from the criterion image in Fig. 2, without (left) and with (right) area closing operator.

Fig. 2 shows the criterion image obtained by applying the method to the boar image. As can be seen in this example, the network appears in dark. However tessellas are not uniform in texture and show local gray-level crests that should be deleted before WT in order to avoid over-segmentation. Following [14], we first compute an area closing [17] of the criterion image which gives fewer minima while retaining crest locations. The WT result is illustrated in Fig. 3. The crest contours now represent correctly the network, which is confirmed by the zoom shown in Fig. 4(a). To determine the width of the network (and not only a one-pixel skeleton as done by WT), which varies through the image, a simple threshold is applied on neighboring pixels of crests: a pixel is aggregated to the crest if its gray value is no more different by $10 \%$ of the skeleton mean gray value. The result of applying such a threshold is shown in Fig. 4(b).

For segmentation, we are now able to only consider the tessellas of the mo- 

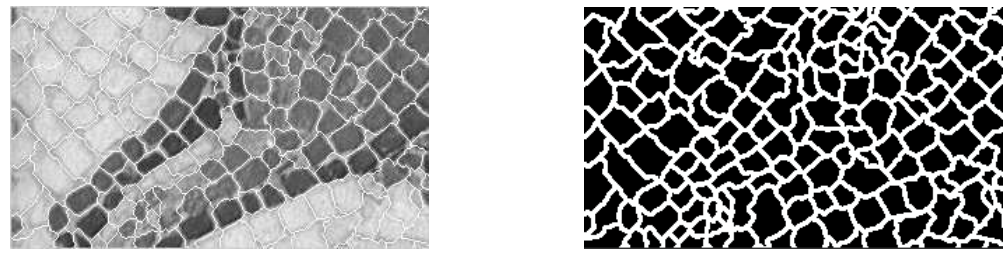

Fig. 4. Result of tessella extraction on the zoom in Fig. 1 (left), and network/tessellas classification (right).

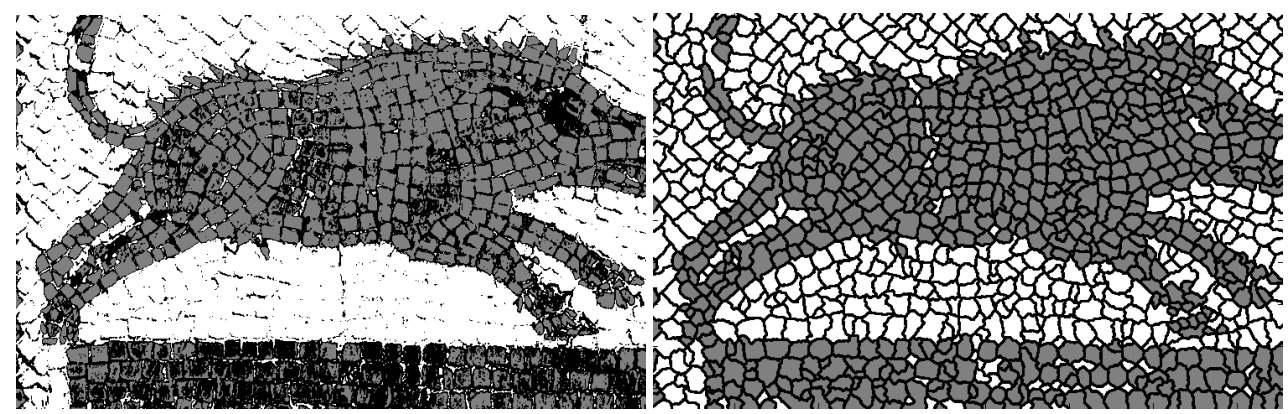

Fig. 5. Segmentation of the mosaic image in Fig. 1 with a pixel-based strategy (left) and the tessella-based strategy proposed here (right), using a k-means algorithm with two classes.

saic, not its network. Moreover, instead of using all pixels from the tessellas, and since tessellas are almost homogeneous in color, we can segment the image by using a tessella-oriented strategy: each tessella is characterized by one or more features used for classification. Simple examples of features are mean gray-level value or variance of the tessella, number of pixels in the tessella, etc. For our application, a simple k-means algorithm on the mean gray-level value of tessellas was sufficient to get a nice segmentation, as illustrated in Fig. 5. This result can be compared with a classical pixel-based k-means strategy. A second segmentation example is given in Fig. 6.

Remark: The entire processing is based on two parameters: (i) the length $l$ of the profile to compute the criterion image and (ii) the area closing threshold $s$. These parameters can be set proportional to the mean tessella size $\alpha$, which is almost constant in a mosaic. Parameter $l$ should be greater than $2 \alpha$ for the 


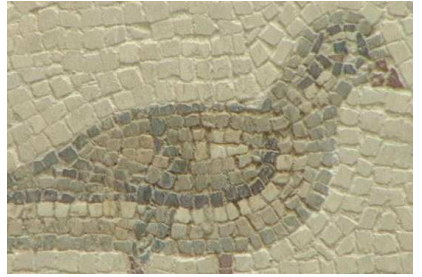

(a)

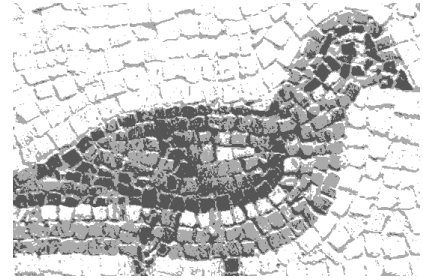

(b)

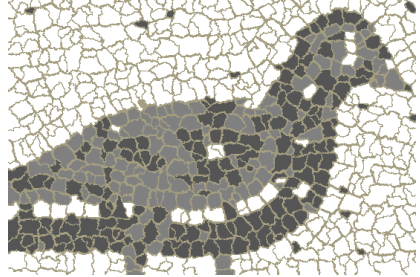

(c)

Fig. 6. Segmentation of a mosaic representing a bird, (a) with a pixel-based strategy (b) and a tessella-based strategy (c), using a k-means algorithm with three classes.

profiles to fit at least two tessellas, and parameter $s$ should be less than $\alpha^{2}$ to avoid small tessellas to be deleted by the morphological operator. In our experiments, values $l=3 \alpha$ and $s=\alpha^{2} / 2$ give good results. Coefficient $\alpha$ depends on the image zoom and can be either set by an operator or estimated automatically on a small uniform part of the mosaic for example.

\section{TESSELLA ORIENTATION ESTIMATION FOR DIRECTIONAL GUIDELINES DETECTION}

Ancient mosaicists avoided to align their tiles according to rectangular grids. Indeed, such grids emphasize only horizontal and vertical lines and may distract the observer from seeing the overall picture. Hence, mosaicists placed tiles in order to emphasis the strong edges of the subject to be represented, influencing the overall perception of the mosaic. Hence, organization and positioning of tessellas are interesting information for experts since they emphasize the main directional guidelines chosen by the artist. This information is of crucial interest for mosaic dedicated applications such as content-based retrieval of mosaic elements or region-based mosaic image compression.

To get directional guidelines, one can first think of using the principal axes of an ellipse-equivalent shape of each tessella, using well-known formulae based 
on geometrical moments (minor an majors axes). However, ancient mosaic tessellas are not box- neither regular-shaped and principal axes quickly appear not enough robust. One major drawback of such method is that the method does not take into account information of neighboring tessellas, which is of great importance for regularization and for recovering the main guidelines that emphasize the "general flow" of a mosaic.

Hence, we propose an energy-based contextual algorithm for retrieving main directional guidelines in a mosaic. The energy to be minimized is constructed by using two key-features: the mean-gray value and borders directions of each tessella. The optimization is done either by gradient descent or by simulated annealing.

\subsection{Methodology}

We denote by $N$ the number of tessellas detected in the mosaic. Each tessella $i$ is represented by

- its barycenter $\left(x_{i}, y_{i}\right)$ computed on the support $\Omega_{i}$ of $i$ :

$$
\left(x_{i}, y_{i}\right)=\left(\frac{m_{1,0}}{m_{0,0}}, \frac{m_{0,1}}{m_{0,0}}\right) \text { with } m_{p, q}=\iint_{\Omega_{i}} x^{p} y^{q} f_{i}(x, y) d x d y
$$

- the list of its neighboring tessellas: $\mathcal{V}_{i}=\left\{v_{i, 1}, \ldots, v_{i, T_{i}}\right\}$. A neighbor is a tessella that shares at least one pixel with $i$.

It should be noted that the number of neighbors $T_{i}$ is different from one tile to the other since tessellas are not organized according to a regular grid.

Each tessella $i$ is characterized by an energy of configuration which links itself to each of its neighbor $v_{i, t} \in \mathcal{V}_{i}$. This energy, denoted by $E_{i, t}, t \in$ $\left[1, \ldots, T_{i}\right]$, is the sum of two complementary terms : 
- The first term $Q$ is based on the mean-gray value of tessellas. It is proportional to the sum of the difference of gray-level means (1) between $i$ and $v_{i, t}$, and (2) between $i$ and the symmetrical tessella of $v_{i, t}$ with respect to $i$. This feature favors alinement of tessellas with low contrast, which is a characteristic of directional guidelines.

- The second term $R$ is based on the orientation of tessella contours. We compute the histogram of the orientation of segments constituting the contour of tessella $i$. This histogram is regularized using a Gaussian kernel, result of which is illustrated in Fig. 7. It should be noted that the two modes at approximatively $90^{\circ}$ each other correspond to the two ambiguous orthogonal main directions of a square-shaped tile. It is then possible to estimate the p.d.f. at angle $\alpha_{i, t}$ given by the barycenter of $i$ and the one of $v_{i, t}$.

Terms $Q$ and $R$ are normalized to belong to range $[0,1]$. We can then initialize the "main direction" of a tile, i.e. the direction of the neighboring tessella which gives the highest $Q+R$ value:

$$
t_{i, \max }=\arg \max _{t \in\left[1, \ldots, T_{i}\right]} E_{i, t} .
$$

The energy of a tessella is then defined as $C_{i}=\left(2-E_{i, t_{i, \max }}\right)+\lambda V_{i}$, with $\lambda$ a weighting factor set manually. Term $V_{i}$ is defined as

$$
V_{i}=\frac{1}{T_{i}} \sum_{t=1}^{T_{i}}\left|\alpha_{i, t_{i, \max }}-\alpha_{t, t_{t, \max }}\right|
$$

which is the normalized sum of absolute difference between the main direction of tile $i$ and the main direction of its neighbor $t$. 


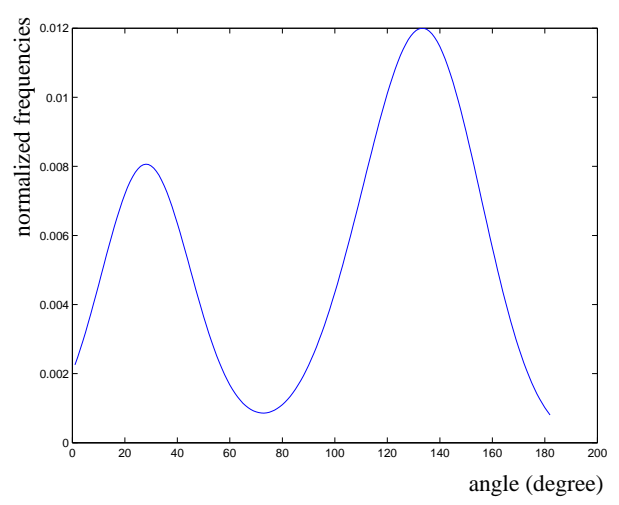

Fig. 7. Plot of the regularized and normalized histogram of the contour orientation of one tile from the boar mosaic in Fig. 1.

We try next to minimize the mosaic energy, defined as the sum of $C_{i}$ for all tessellas in the mosaic. This is done by selecting the tessella $i$ which gives the highest value for $V_{i}$. To reduce the contribution of this tessella, we try another main direction and recompute the mosaic energy. At that point, two strategies have been tested:

- Deterministic framework (Gradient Descent): if the mosaic energy reduces then the new main direction is validated, otherwise another main direction is tested. When all directions for this tile have been tested, we repeat the process for the next tile with high $V_{i}$ value.

- Stochastic framework (Simulated Annealing): a configuration which gives a higher mosaic energy can be validated according to the simulated annealing principle [18]. This strategy allows to search for the global minimum, which can not be reached with previous strategy since the mosaic energy function is not convex.

The process is iterated until the mosaic energy is almost constant. The cartography of tessella orientation is made of the main direction of each tile at the last iteration. 


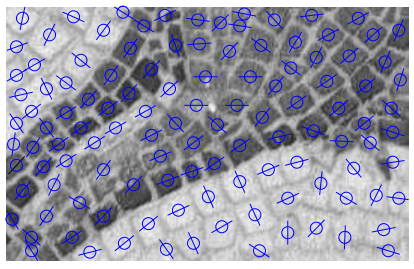

(a) Configuration at initialization

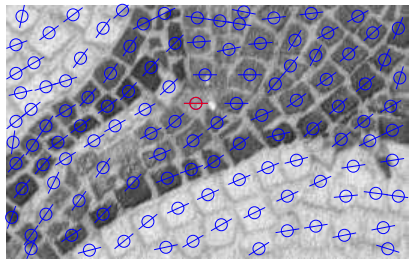

(b) Configuration after SA

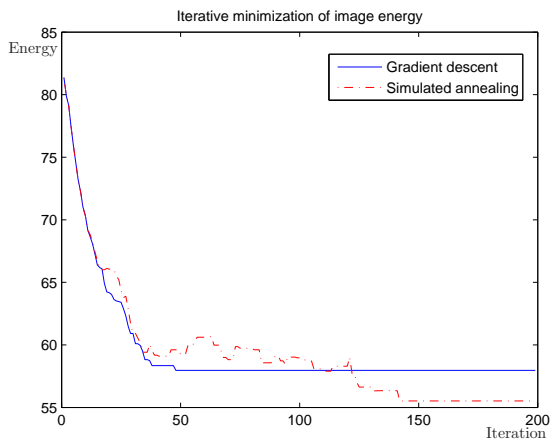

(c) Energy evolution for GD and SA

Fig. 8. Cartography of tessellas orientation. Each tessella is characterized by its center of mass (circle) and its orientation (segment crossing the circle).

\subsection{Experimental results}

Fig. 8 illustrates the application of the tessella orientation methodology on the zoom of the boar image in Fig. 1. From the initial configuration of tessellas (a) we get the final configuration (b) using Simulated Annealing (SA) for optimization. Figure (c) shows the evolution of the computed energy during iterations of both gradient descent (GD) algorithm and simulated annealing one. As expected, SA reaches a lower minimum than GD but to the detriment of numerous additional iterations (150 for SA versus 50 for GD). Indeed, GD searches for a local minimum and is highly dependent on the initial configuration, whereas SA is expected to reach the global minimum due to its stochastic nature.

The tessella cartography obtained with SA optimization is really satisfying when visually compared to the main directional guidelines of the mosaic. This 


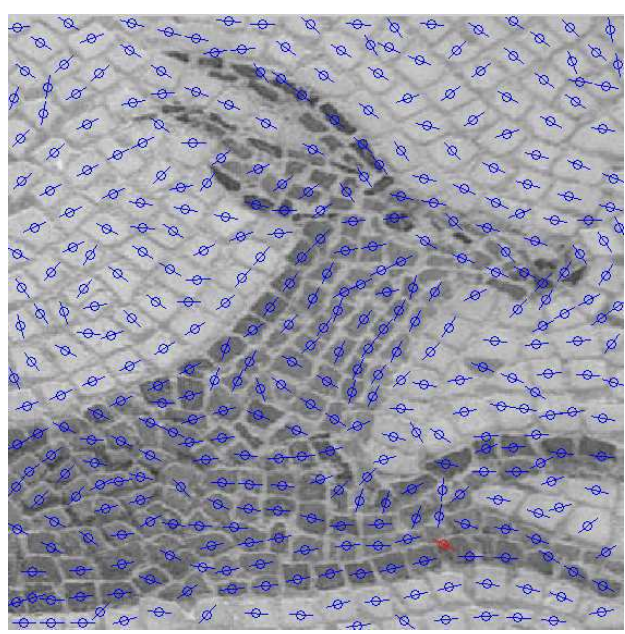

(a) Configuration after SA

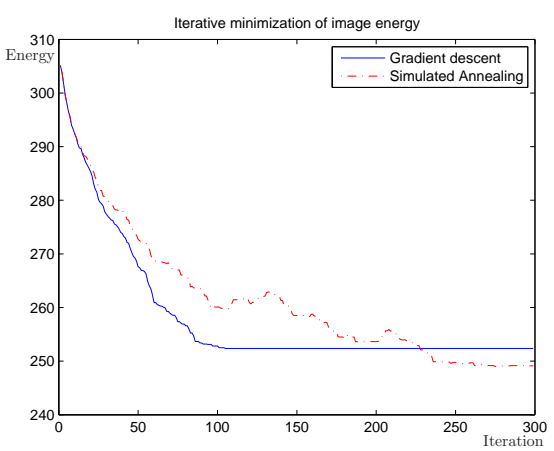

(b) Energy evolution for GD and SA

Fig. 9. Another example of tessellas orientation cartography.

is especially true for regions at the borders between classes. A second example of cartography is proposed in Fig. 9. Once again, the tessella orientation estimation methodology, which makes use of contextual information, gives regularized results that emphasize the mosaic guidelines. Nevertheless, confusions can be found on areas with homogeneous colors and where tessellas are squarelike shaped. Indeed, for those kinds of tessellas two orthogonal directions are equally probable, which generally gives ambiguous results. However, these areas of uniform color are of limited interest for object-based scene applications, such as mosaic pattern recognition.

Figure 10 shows a failure case in tessella orientation estimation. Indeed, the result will not allow to find the main directional guidelines in the mosaic, that can be more easily observed in Fig. 6(a). The main reason comes from an overdetection of tessellas from the extraction step. This behavior is observed on mosaics built with tessellas of different sizes (e.g. large tessellas for the background and small ones for objects or details). Hence the shape of extracted tiles does not correspond to the shape of tessellas and the orientation is corrupted, 


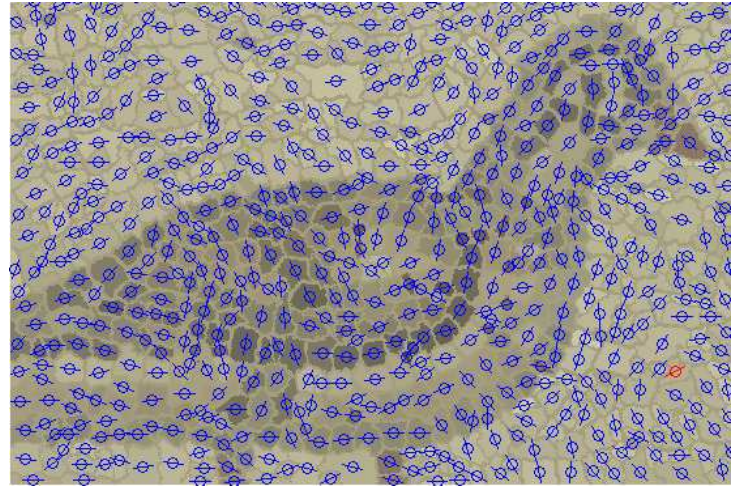

Fig. 10. Result of guidelines detection for the "bird" mosaic in Fig. 6.

showing no particular guideline in the mosaic.

Remark: For all experiments, weighting factor $\lambda$ has been set to 1. A study not reported here showed the low impact of $\lambda$ value on the results.

\section{CONCLUSION}

In this work, a method for analyzing structure and color of ancient mosaic images has been presented, based on a tile-oriented strategy. To extract tessellas from the cement network, we applied a watershed transform on a criterion image computed from the original one. The criterion image was generated in order to exhibit the cement network as watershed crests and each tessella as a catchment basin. Then, from the individual tiles, we were able to compute a tessella-based k-means classification, using the mean-gray value as a feature to characterize tessellas. Results of segmentation are distinctly of higher quality than those obtained from a pixel-based k-means strategy.

Then, in order to help archeologists understand mosaic structure and mosaicists' way of working, and also to facilitate extraction of individual semantic objects in complex mosaic scenes, we proposed a method to estimate the main directional guidelines of tessellas in mosaics. To that goal, each tessella is described by a contextual energy computed from the mean-gray value and 
the main directions of tile borders. The minimum energy is searched for by means of the simulated annealing algorithm. Results showing cartographies of tessellas orientation are really interesting since most of searched guidelines are retrieved, especially those at the borders between objects and scene background.

These processings are first steps toward a system devoted to the indexation and retrieval of sematic objects in mosaic images, which should help archeologists to compare mosaics from different sites or built at different dates. Future works will include a tessella-based invariant description of objects to enable the comparison between images of mosaic taken at different zooms and orientations for example. Image compression of ancient mosaics, using a tessella-based coding strategy, is also an interesting perspective, e.g. for quick look at a distant catalog using Internet.

\section{References}

[1] S. Battiato, G. D. Blasi, G. M. Farinella, and G. Gallo, "Digital mosaic frameworks: an overview," Computer Graphic Forum 26, 794-812 (2007).

[2] M. M'hedhbi, R. Mezhoud, S. M'hiri, and F. Ghorbel, "A new content-based image indexing and retrieval system of mosaic images," in Third Int. Conf. on Information and Communication Technologies: from Theory to Applications (ICTTA'06), 1715-1719, (Damascus, Syria) (2006).

[3] F. Ghorbel, "A complete invariant description for gray-level images by the harmonic analysis approach," Pattern Recognition Letters 15, 1043-1051 (1994).

[4] S. Derrode and F. Ghorbel, "Robust and efficient Fourier-Mellin transform approximations for invariant grey-level image description and reconstruction," Computer Vision and Image Understanding 83, 57-78 (2001). 
[5] H. Mayer, S. Hinz, U. Bacher, and E. Baltsavias, "A test of automatic road extraction approaches," in Photogrammetric Computer Vision (PCV'06), (Bonn, Germany) (2006).

[6] J. B. Mena and J. A. Malpica, "An automatic method for road extraction in rural and semi-urban areas starting from high resolution satellite imagery," Pattern Recognition Letters 26, 1201-1220 (2005).

[7] A. C. S. Chung, "Image segmentation methods for detecting blood vessels in angiography," in IEEE Int. Conf. on Automation, Robotics and Computer Vision (ICARCV'06), 1-6, (Singapore) (2006).

[8] P. J. Yim, G. Boudewijn, C. Vasbinder, V. B. Ho, and P. L. Choyke, "Isosurfaces as deformable models for magnetic resonance angiography," IEEE trans. on Medical Imaging 22, 875-881 (2003).

[9] M. A. Fischler, J. M. Tenenbaum, and H. C. Wolf, "Detection of road and linear structures in low resolution aerial images using multi-source knowledge integration techniques," Computer Graphics and Image Processing 15(3), 201-223 (1981).

[10] C. Steger, "An unbiased detector of curvilinear structures," IEEE trans. on Image Processing 20, 113-125 (1998).

[11] A. Baumgartner, S. Hinz, and C. Wiedemann, "Efficient methods and interfaces for road tracking," in Photogrammetric Computer Vision (PCV'02), B:28, (Graz, Austria) (2002).

[12] O. Wink, W. Niessen, and M. Viergever, "Multiscale vessel tracking," IEEE trans. on Medical Imaging 23, 130-133 (2004).

[13] F. Tupin, H. Maitre, J. F. Mangin, J. M. Nicolas, and E. Pechersky, "Detection of linear features in SAR images: application to road network extraction," IEEE trans. on Geoscience and Remote Sensing 36(2), 434-453 (1998). 
[14] T. Géraud and J. B. Mouret, "Fast road network extraction in satellite images using mathematical morphology and Markov random fields," EURASIP Journal on Applied Signal Processing 2004(16), 2503-2514 (2004).

[15] C. M. van Bemmel, L. J. Spreeuwers, M. A. Viergever, and W. J. Niessen, "Levelset-based artery-vein separation in blood pool agent CE-MR angiograms," IEEE trans. on Medical Imaging 22, 1224-1234 (2003).

[16] M. Rochery, I. H. Jermyn, and J. Zerubia, "Higher order active contours," Int. J. of Computer Vision 69, 27-42 (2006).

[17] L. Vincent, "Grayscale area openings and closings: their applications and efficient implementation," in Proc. EURASIP Workshop on Mathematical Morphology and its Applications to Signal Processing, 22-27, (Barcelona, Spain) (1993).

[18] S. Kirkpatrick, C. D. Gelatt, and M. P. Vecchi, "Optimization by simulated annealing," Science, New Series 220, 671-680 (1983). 


\section{List of Figures}

1 Excerpt of an ancient mosaic showing a boar with a zoom on its hind legs. This image will be used later as a guiding thread for algorithm illustration. . . . . . . . . . . . . 3

2 Criterion image obtained from the boar image in Fig. 1. . . . . . 6

3 Extraction of tessellas from the criterion image in Fig. 2, without (left) and with (right) area closing operator. . . . . . . 6

4 Result of tessella extraction on the zoom in Fig. 1 (left), and network/tessellas classification (right). . . . . . . . . 7

5 Segmentation of the mosaic image in Fig. 1 with a pixel-based strategy (left) and the tessella-based strategy proposed here (right), using a k-means algorithm with two classes. . . . . . . . . . 7

6 Segmentation of a mosaic representing a bird, (a) with a pixelbased strategy (b) and a tessella-based strategy (c), using a kmeans algorithm with three classes. . . . . . . . . . 8

$7 \quad$ Plot of the regularized and normalized histogram of the contour orientation of one tile from the boar mosaic in Fig. 1. . . . . . . 11

8 Cartography of tessellas orientation. Each tessella is characterized by its center of mass (circle) and its orientation (segment crossing the circle). . . . . . . . . . . . . 12

9 Another example of tessellas orientation cartography. . . . . . . 13

10 Result of guidelines detection for the "bird" mosaic in Fig. 6. . . 14 


\section{Biographies}

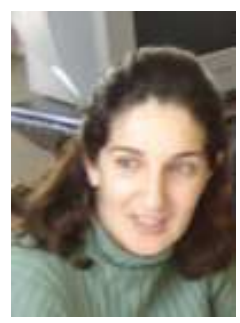

Lamia Benyoussef: L. Benyoussef received the electronics and informatics engineering degree from the department of computer science of the University of Sciences, Tunis, Tunisia in 1996. She received her Ph.D. degree from Bordeaux University, France, in signal and image processing in 2004. From 2003 to 2007, she has been with the College of higher education of Sciences and Technics of Tunis (ESSTT) and GRIFT department of École Nationale des Sciences de l'Informatique, Tunisia, as Associate Professor. Since September 2007, she is currently an Associate Professor in the GSM Group, Institut Fresnel (CNRS UMR 6133), Marseille, France. Her research interests include group theoretical image representation, registration methods and Markov models for segmentation.

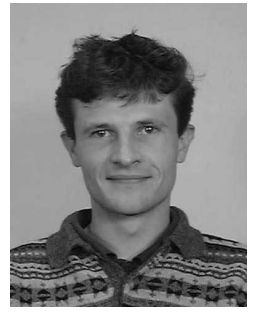

Stéphane Derrode: S. Derrode received the telecommunication engineering degree from Télécom Lille 1, France, in 1995, and his $\mathrm{PhD}$ degree from the University of Rennes I, France, in 1999. From 1999 to 2001, he worked as a 
research engineer in the École Nationale Supérieure des Télécommunications de Bretagne (ITI department), Brest (France). Since September 2001, he has been with the École Centrale Marseille, where he is currently an Associate Professor in the GSM Group, Institut Fresnel (CNRS UMR 6133). His research interests include invariance, group theoretical image representation, and Markov models for pattern recognition, image indexing and segmentation. 